\title{
A new neutrophil candida killing test: chromium-51 release from Candida guilliermondii
}

\author{
S MARTIN, ATM GHONEIM, AND JA CHILD \\ From the Department of Microbiology, University of Leeds and Department of Haematology, Leeds \\ General Infirmary, UK
}

SUMMARY Neutrophil candidacidal function was measured by a chromium-51 $\left({ }^{51} \mathrm{Cr}\right)$ release technique, using Candida guilliermondii as the test organism. The effect of varying the neutrophil:candida ratio, the time course of ${ }^{51} \mathrm{Cr}$ release, the effect of different serum concentrations, and the reproducibility of the technique were investigated. Results from 33 experiments, using neutrophils from 35 normal donors and six patients with known neutrophil defects, show the technique to be a reliable and reproducible assay of candida killing. The chromium release assay has the advantage of being easy to perform and not liable to subjective errors.

A number of in vitro tests have been developed to assess the ability of neutrophils to kill microorganisms.

One technique that is widely used depends upon microscopic evaluation of stained smears prepared from neutrophil-candida suspensions. ${ }^{1-3}$ As well as being tedious to perform, such techniques are liable to subjective errors. Ingested organisms may sometimes be neither clearly viable nor yet definitely non-viable 'ghosts'. When one experienced individual is performing the assay the results may be comparable. However, the possibilities of between observer error are considerable. Yamamura et al. ${ }^{4}$ described a method in which release of ${ }^{51} \mathrm{Cr}$ from labelled Candida albicans was used to determine the ability of neutrophils to kill that organism. The release of radioactivity correlated with the number of candida killed, as shown by methylene blue dye exclusion. The disadvantage of using $C$. albicans is that at candida:neutrophil ratios of greater than 1:1 it tends to grow into hyphal forms with in the neutrophils, thus disrupting them. ${ }^{256}$ It is felt that the candidacidal function of a neutrophil population cannot be adequately assessed until each neutrophil has ingested some candida. This is achieved only at candida:neutrophil ratios of $3: 1$ or greater.

This paper describes modifications to the chromium release assay which allow Candida guilliermondii to be used in preference to $C$. albicans. $C$. guilliermondii remains in the yeast form, making it possible to employ higher candida:neutrophil ratios, ${ }^{27}$ and the use of this species in conventional

Received for publication 10 January 1980 non-isotopic candida killing tests has proven very valuable, notably in the assessment of neutrophil function in patients with haematological malignancies. ${ }^{12} 89$ The level of ${ }^{51} \mathrm{Cr}$ release obtained is compared with the percentage of candida killed, as determined microscopically.

\section{Material and methods}

NEUTROPHIL PREPARATION

Heparinised blood samples were sedimented in dextran $(6 \% \mathrm{w} / \mathrm{v}$ in $0.9 \%$ saline $)$ at $37^{\circ} \mathrm{C}$ for 45 to 60 minutes.

Neutrophils were separated from the leucocyte rich plasma by centrifugation for 25 minutes at $400 \mathrm{~g}$ over Ficoll-Triosil, specific gravity $1 \cdot 077 . .^{10}$ The pellet was washed twice in Hanks's balanced salt solution (HBSS) containing $2 \mathrm{U} / \mathrm{ml}$ preservative-free heparin, and re-suspended to $5 \times 10^{6}$ neutrophils $/ \mathrm{ml}$. The leucocytes were $>90 \%$ neutrophils and the viability by trypan blue dye exclusion $>95 \%$.

\section{SERUM}

Pooled AB serum from healthy blood donors was used in all assays. The serum was stored in $1 \mathrm{ml}$ aliquots at $-20^{\circ} \mathrm{C}$.

\section{CANDIDACIDAL ASSAY}

The method has been described previously by El-Maalem and Fletcher ${ }^{2}$ and Child et al. ${ }^{1}$ The assay mixture comprised $0.05 \mathrm{ml} \mathrm{AB}$ serum, $0.25 \mathrm{ml}$ neutrophils $\left(5 \times 10^{6} / \mathrm{ml}\right), 0 \cdot 1 \mathrm{ml} C$. guilliermondii $\left(3 \times 10^{7} / \mathrm{ml}\right), 0 \cdot 1 \mathrm{ml}$ HBSS-gel, to give a final candida:neutrophil ratio of $3: 1$. Aliquots were 
removed after incubation for 60 minutes at $37^{\circ} \mathrm{C}$, and smears were prepared and stained with MayGrünwald Giemsa. The number of candida within 100 neutrophils were counted for viable and nonviable organisms.

CHROMIUM LABELLING OF CANDIDA

GUILLIER MONDII

C. guilliermondii was subcultured in Sabouraud's broth for three days at $30^{\circ} \mathrm{C}$. Five millilitres of the candida suspension (adjusted to $2.5 \times 10^{7}$ organisms/ $\mathrm{ml}$ ) were centrifuged at $700 \mathrm{~g}$ for 10 minutes, and the cell pellet was mixed gently with $100 \mu \mathrm{Ci}{ }^{51} \mathrm{Cr}$, specific activity $1 \mathrm{mCi} / \mathrm{ml}$ (Radiochemical Centre, Amersham). After 120 minutes' incubation at $37^{\circ} \mathrm{C}$ the candida were washed twice and then re-suspended in $5 \mathrm{ml}$ phosphate-buffered saline (PBS). This suspension could be stored for two weeks at $4^{\circ} \mathrm{C}$. Before use the candida were washed once in PBS and re-suspended in HBSS containing $0.1 \%$ gelatin (HBSS-gel) to $7.5 \times 10^{6} / \mathrm{ml}$.

\section{CHROMIUM RELEASE ASSAY}

The following parameters were investigated:

1 Effects of varying the neutrophil:candida ratio in order to determine the optimum ratio for use in function tests.

2 Time course of chromium release so that a suitable incubation time could be selected for use in the standard assay.

3 The effect of different serum concentrations on chromium release.

4 Variation between replicates, to test the reproducibility of the technique.

5 Demonstration of defects in neutrophil function in patients with known functional abnormalities.

6 Comparison of chromium release and percentate killing of candida by neutrophils.

Unless otherwise stated, standard assay conditions were $0.1 \mathrm{ml}$ neutrophils $\left(5 \times 10^{6} / \mathrm{ml}\right), 0.2 \mathrm{ml} C$. guilliermondii $\left(7.5 \times 10^{6} / \mathrm{ml}\right), 0.15 \mathrm{ml}$ HBSS-gel, and $0.05 \mathrm{ml}$ serum. Each test was set up in RT30 plastic tubes (Sterilin) in triplicate and incubated for 60 minutes at $37^{\circ} \mathrm{C}$ on an orbital shaker $(150 \mathrm{rpm})$. After incubation $0.2 \mathrm{ml}$ DNase $(0.1 \mathrm{mg} / \mathrm{ml})$ and $0.1 \mathrm{ml}$ sodium deoxycholate $(24 \mathrm{mg} / \mathrm{ml})$ were added, and the mixture was incubated for a further 15 minutes at $37^{\circ} \mathrm{C}$. The tubes were then centrifuged at $2000 \mathrm{~g}$ for 10 minutes; $0.2 \mathrm{ml}$ of the supernatants was collected and counted in a Packard gamma counter.

The results were expressed by the following formula:

$$
\% \text { release }=\frac{(X-S)}{(T-S)} \times 100
$$

where $X$ is the radioactivity released in the presence of neutrophils, $S$ is the spontaneous release after incubation in medium, and $T$ is the total amount of radioactivity in $0.2 \mathrm{ml}$ of the final candida suspension without centrifugation.

\section{Results}

EFFECT OF VARYING THE NEUTROPHIL: CANDIDA RATIO

Figure 1 shows the percentage of chromium release obtained from six experiments in which a constant number of neutrophils $\left(5 \times 10^{5}\right)$ were incubated with $5 \times 10^{5}$ to $32 \times 10^{6}$ candida to give ratios of $1: 1$ to $1: 64$. Between the ratios $1: 1$ to $1: 4$ the percentage of chromium released was constant, but for the higher ratios there was a decrease in the percentage release. For equal numbers of neutrophils and candida, or slight candida excess, the neutrophils were readily able to kill and digest the yeast. A larger number of candida stressed the neutrophils so that a lower percentage of organisms were destroyed.

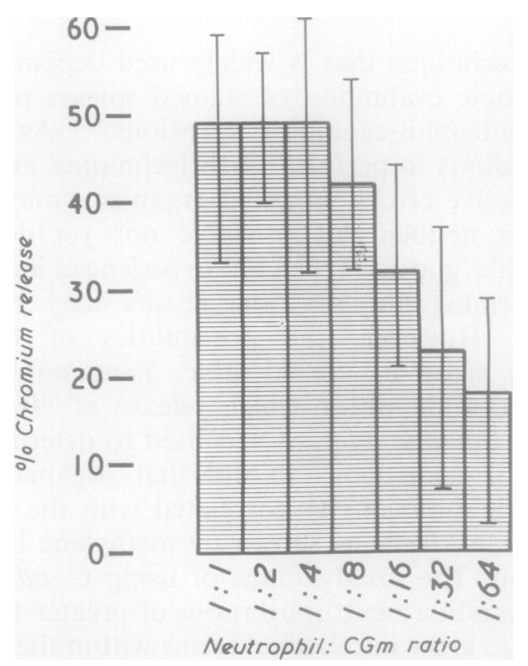

Fig. 1 Percentage chromium release after 60 min for different neutrophil:candida ratios. $5 \times 10^{5}$ neutrophils were incubated with $5 \times 10^{5}$ to $32 \times 10^{6}$ candida: results of six experiments. $C G M=$ Candida guilliermondii. Mean + range.

Microscopic examination showed that with a ratio of $1: 3$ every neutrophil ingested one or more candida while none became excessively full. As this ratio was also on the constant portion of the curve, a neutrophil:candida ratio of $1: 3$ was chosen for subsequent tests. 
TIME COURSE OF CHROMIUM RELEASE

Figure 2 demonstrates four experiments in which supernatants were harvested at different times. A 60-minute incubation time was chosen for subsequent assays. At this time the amount of chromium released was sufficient for accurate measurements. There was no advantage in longer incubation because after 60 minutes the amount of chromium released began to plateau.

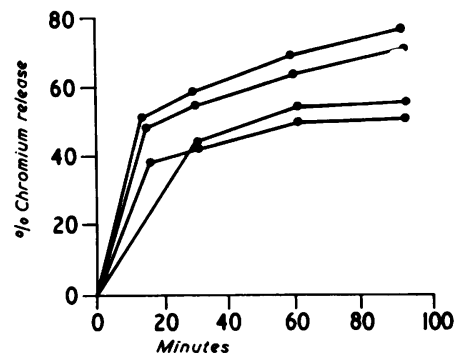

Fig. 2 Time course for ${ }^{51} \mathrm{Cr}$ release: results of four experiments using neutrophils from normal controls.

\section{EFFECT OF DIFFERENT SERUM}

CONCENTRATIONS

Table 1 shows the results of six experiments in which the effect of different serum concentrations in the chromium release assay was investigated. Doubling dilutions of serum from $20 \%$ to $1.25 \%$ were used in addition to a control without serum. All of the serum concentrations studied induced a similarly low level of spontaneous chromium release from labelled candida when compared with the spontaneous release from candida in the absence of serum.

Table $1{ }^{51} \mathrm{Cr}$ release assay: effect of different serum concentrations on ${ }^{51} \mathrm{Cr}$ release from labelled candida. Mean of six experiments

\begin{tabular}{lll}
\hline$\%$ serum & \multicolumn{2}{l}{ Percentage ${ }^{51}$ Cr release from C. guilliermondii } \\
\cline { 2 - 3 } & $S$ & $P M N$ \\
\hline 0 & - & $5 \cdot 7$ \\
1.25 & 1.4 & 27 \\
2.5 & 1.8 & 33 \\
5 & 1.4 & 36 \\
10 & 1.0 & 37 \\
20 & 0.7 & 36 \\
\hline
\end{tabular}

$\mathbf{S}=$ spontaneous release compared to control without serum; PMN = neutrophil induced release.

Neutrophil induced chromium release from labelled candida increased with the amount of serum present up to a concentration of $5 \%$. When the serum concentration was between $5 \%$ and $20 \%$ the level of neutrophil induced chromium release was constant.
A serum concentration of $10 \%$ was chosen for use in the standard assay to ensure that the amount of chromium released from candida in the presence of neutrophils would be independent of the serum.

\section{VARIATION BETWEEN REPLICATES}

Table 2 shows the variation in the amount of chromium released from replicates in three experiments. Neutrophils from a different normal control donor were used for each experiment. The results showed little variation, confirming that the sampling technique was consistent.

Table $2{ }^{51} \mathrm{Cr}$ release assay: variation between replicate assays using neutrophils from normal controls in three experiments

\begin{tabular}{lllll}
\hline Experiment & $\begin{array}{l}\text { Number of } \\
\text { replicates }\end{array}$ & Mean cpm & $S D(\mathrm{cpm})$ & $S D \%$ \\
\hline 1 & 16 & 2664 & \pm 85 & \pm 3 \\
2 & 12 & 1248 & \pm 65 & \pm 5 \\
3 & 8 & 1188 & \pm 42 & \pm 3 \\
\hline
\end{tabular}

DEMONSTRATION OF DEFECTS IN

NEUTROPHIL FUNCTION

Table 3 shows the amount of chromium released and the corresponding percentage candida killing for various patients who were known to have defects in their neutrophil function. There were two patients with chronic granulomatous disease (CGD), two with preleukaemia, and one with advanced Hodgkin's disease.

Table $3{ }^{31} \mathrm{Cr}$ release and $\%$ candida killing for normal controls and five patients with defective neutrophil

function

\begin{tabular}{lll}
\hline Disease & $\%{ }^{51}$ Cr release & $\%$ killing \\
\hline Control & 53 & 66 \\
CGD 1 & 1 & 13 \\
Control & 51 & 64 \\
CGD 2 & 20 & 32 \\
Control & 52 & 56 \\
Preleukaemia 1 & 31 & 39 \\
Control & 55 & 59 \\
Preleukaemia 2 & 21 & 47 \\
Control & 53 & 63 \\
Hodgkin's disease & 37 & 48 \\
\hline
\end{tabular}

CGD $=$ chronic granulomatous disease

COMPARISON OF CHROMIUM RELEASE AND PERCENTAGE KILLING

In 33 experiments using neutrophils from 35 normal donors a range of $33-60 \%$ chromium release was observed (mean $49 \pm 5 \%$ ), the corresponding range for percentage killing, as detected microscopically, being $42-79$ (mean $60 \pm 11) \%$.

Figure 3 shows the correlation between chromium 


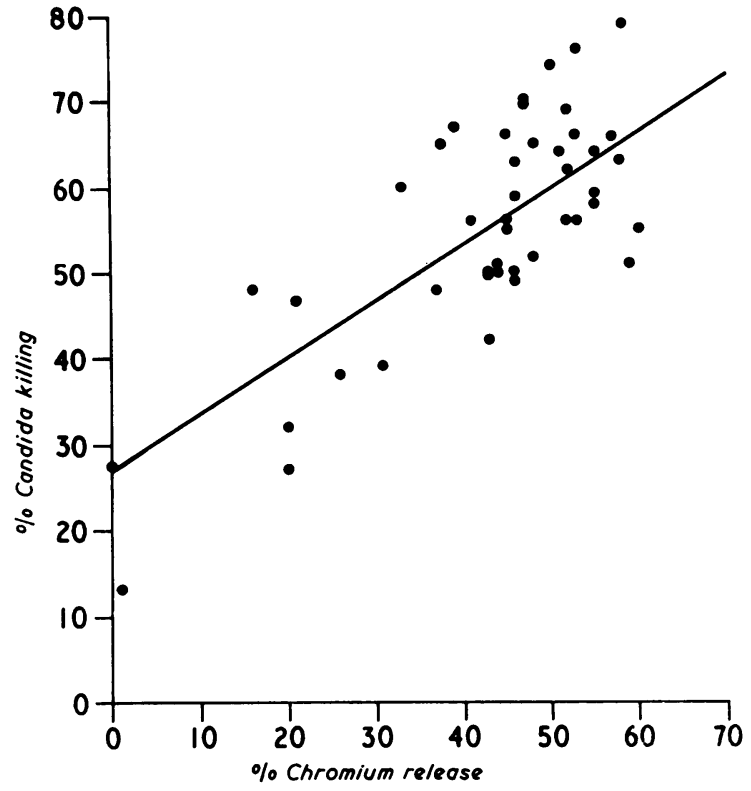

Fig. 3 Regression lines and correlation between $\%$ candida killing, as determined microscopically, and \% chromium released: results of 33 experiments using normal and defective neutrophils: $r=0.72 ; \mathrm{P}<0.001$.

release and candida killing for data accumulated from the above-mentioned controls, two children with CGD, three patients with preleukaemia, and one with Hodgkin's disease: correlation coefficient $\mathrm{r}=0.72, \mathrm{P}<0.001$.

\section{Discussion}

The majority of methods previously used for measuring the candidacidal activity of leucocytes have depended upon microscopic evaluation of stained or unstained organisms. Yamamura et al. ${ }^{4}$ were the first to describe a chromium release technique for the measurement of candidacidal activity of leucocytes. The ${ }^{51} \mathrm{Cr}$ released for phagocytosed labelled C. albicans could be detected in the medium, and the amount of that chromium was found to reflect the loss in viability of the organisms. In practice this test probably has limitations in that at neutrophil:candida ratios greater than $1: 1, C$. albicans tends to grow into hyphal forms with disruption and death of the neutrophils. Germ tube formation by $C$. albicans in the presence of serum can become evident within one hour. ${ }^{6}$ Furthermore, there have been recent suggestions that $C$. albicans can produce a substance which interferes with neutrophil phagocytic function. ${ }^{11}$ When neutrophil killing function per se is under investigation, there is an obvious advantage in using an organism which does not interfere with phagocytosis or mask function by disrupting the phagocyte.

By adapting the chromium release assay, as described here for $C$. guilliermondii, it was possible to employ a neutrophil:candida ratio of $1: 3$, which ensured that every neutrophil ingested at least one organism. It seems probable that only when all the neutrophils have ingested some organisms can their function be adequately tested, which is not necessarily the case with a ratio of $1: 1$. A normal range was established for the amount of ${ }^{51} \mathrm{Cr}$ released from the candida in the presence of neutrophils from healthy donors. This range was $33-60 \%$ distributed normally about a mean of $49 \%$ with a standard deviation of $5 \%$. This range is similar to that obtained by Yamamura et al. using C. albicans. ${ }^{4}$ The standard deviation found here for the percentage chromium release $( \pm 5 \%)$ was less than for percentage killing as determined by the microscopic test $( \pm 11 \%)$. The chromium release technique would, therefore, seem to be at least as reliable as the microscopic technique and has the advantage of not being liable to serious subjective errors.

In order to evaluate the test further, patients known to have neutrophil defects were studied.

Children with CGD are known to have a gross defect in their neutrophil oxidative metabolism. ${ }^{12}$ They also have a defect in their ability to kill candida. The results presented in Table 3 show that the neutrophils from our two patients with CGD killed $13 \%$ and $32 \%$ of the added candida respectively as compared with $66 \%$ and $64 \%$ killed by normal controls. The corresponding amounts of chromium released were $1 \%$ and $20 \%$ for the patients and $53 \%$ and $51 \%$ for the control.

Table 3 also shows the reduced candida killing for two patients with preleukaemia and one with advanced Hodgkin's disease as compared with normal controls. There was a corresponding reduction in the percentage of chromium released from labelled candida. Defective candida killing in some patients with preleukaemia and Hodgkin's disease has been previously described. ${ }^{113}$

The findings in CGD, preleukaemia, and Hodgkin's disease illustrated that the chromium release technique, in its modified form, was capable of detecting defects in neutrophil function.

The correlation curve shows that there is good positive correlation between levels of chromium released and the number of candida killed. However, the regression line suggests that for low levels of candida killing there is no detectable chromium release. This could be explained by the fact that the majority of points on the graph are for fairly high 
values of killing and chromium release.

The values recorded for percentage killing tended to be slightly higher than the percentage chromium released. After killing has occurred, some digestion of the candida by the neutrophils is probably necessary before maximum levels of chromium release are obtained.

The disadvantage of any technique employing radiochemicals is that they are expensive to purchase and require the use of specialised equipment. However, this is an assay which is objective, reproducible, easy to perform, and needs only a small amount of blood. Results can be obtained in 24 hours, more rapidly than is the case with the microscopic examination of a large number of samples in the non-isotopic methods. Where appropriate facilities are available it should be feasible to use this technique for routine screening of patients for defects in candidacidal function.

Mrs S Martin is supported by grants from the Special Trustees, Leeds General Infirmary, and Janssen Pharmaceutical Ltd. We are also grateful for the encouragement and support of Professor EM Cooke, Dr BE Roberts, and Professor G Gowland.

\section{References}

1 Child JA, Martin S, Cawley JC, Ghoneim ATM. Defective microbicidal function of neutrophils in haematological malignancies and lymphomas. Correction by levamisole in vitro. Biomedicine 1978;29:159-61.

${ }^{2}$ El-Maalem H, Fletcher J. Defective neutrophil function in chronic granulocytic leukaemia. Brit J Haemat 1976;34: 95-103.

${ }^{3}$ Lehrer RI. Measurement of candidacidal activity of specific leukocyte types in mixed cell populations. Normal, myeloperoxidase-deficient and chronic granulomatous disease neutrophils. Infect Immun 1970;2:42-7.

4 Yamamura M, Boler J, Valdimarsson H. A ${ }^{51}$ Chromium release assay for phagocytic killing of Candida albicans. J Immunol Meth 1976;13:227-33.

${ }^{5}$ Rosner F, Valmont I, Kozinn PJ, Caroline L. Leukocyte function in patients with leukaemia. Cancer 1970;25: 835-42.

- Chilgren RA, Hong R, Quie PG. Human serum interactions with Candida albicans. J Immunol 1968;101:128-32.

${ }^{7}$ Goldstein E, Grieco MH, Finkel G, Louria DB. Studies on the pathogenesis of experimental Candida parapsilosis and Candida guilliermondii infections in mice. J Infect Dis 1965;115:293-302.

${ }^{8}$ El-Maalem H, Fletcher J. Defective hydrogen peroxide production in chronic granulocytic leukaemia neutrophils. Brit J Haemat 1979;41:49-55.

${ }^{9}$ Child JA, Cawley JC, Martin S, Ghoneim ATM. Microbicidal function of the neutrophils in hairy cell leukaemia. Acta Haemat 1979;62:191-8.

${ }^{10}$ Boyum A. Separation of leucocytes from blood and bone marrow. Scan J Clin Lab Invest 1968;21(Suppl):97.

${ }_{11}$ Diamond RD, Krzesicki R, Oppenheim F. Inhibition of phagocytic function of human neutrophils by a substance released from Candida albicans hyphae. Proceedings of the 11th International Congress of Chemotherapy: Boston 1979: publ. Am Soc Microbiol Washington. In press.

12 Holmes B, Park BH, Good RA. Studies of the metabolic activity of leukocytes from patients with a genetic abnormality of phagocytic function. J Clin Invest 1967; 46:1422-32.

${ }^{13}$ Houghton RI. Neutrophil function and diagnosis of preleukaemic states. Postgrad Med J 1979;55:396-9.

Requests for reprints to: Dr JA Child, Department of Haematology, The General Infirmary at Leeds, Great George Street, Leeds LS1 3EX, UK. 